Article

\title{
Economic impact of SARS-COV2 infection in the Department of diagnostic imaging
}

\author{
Massimo Cristofaro ${ }^{1 *}$, Ada Petrone ${ }^{2}$, Fabrizio Albarello ${ }^{3}$, Federica Di Stefano ${ }^{4}$, Nicoletta Fusco ${ }^{5}$, Vincenzo \\ Schininà ${ }^{6}$, Stefania Ianniello ${ }^{7}$, Paolo Campioni ${ }^{8}$.
}

1 Lazzaro Spallanzani, National Institute for Infectious Diseases - IRCCS, Via Portuense, 292, cap 00148 Rome, Italy; massimo.cristofaro@inmi.it

2 Lazzaro Spallanzani, National Institute for Infectious Diseases - IRCCS, Via Portuense, 292, cap 00148 Rome, Italy; ada.petrone@inmi.it

3 Lazzaro Spallanzani, National Institute for Infectious Diseases - IRCCS, Via Portuense, 292, cap 00148 Rome, Italy; fabrizio.albarello@inmi.it

4 Lazzaro Spallanzani, National Institute for Infectious Diseases - IRCCS, Via Portuense, 292, cap 00148 Rome, Italy; federica.distefano@inmi.it

5 Lazzaro Spallanzani, National Institute for Infectious Diseases - IRCCS, Via Portuense, 292, cap 00148 Rome, Italy; nicoletta.fusco@inmi.it

6 Lazzaro Spallanzani, National Institute for Infectious Diseases - IRCCS, Via Portuense, 292, cap 00148 Rome, Italy; vincenzo.schinina@inmi.it

7 Lazzaro Spallanzani, National Institute for Infectious Diseases - IRCCS, Via Portuense, 292, cap 00148 Rome, Italy; stefania.janniello@inmi.it

8 Lazzaro Spallanzani, National Institute for Infectious Diseases - IRCCS, Via Portuense, 292, cap 00148 Rome, Italy; paolo.campioni@inmi.it

* Correspondence: massimo.cristofaro@inmi.it; Tel.: +390655170358

\begin{abstract}
The COVID-19 pandemic caused an unprecedented effect on national radiological investigations. Since the World Health Organization officially declared the COVID-19 as a global pandemic, health policies have been rapidly organized to limit the spread of the virus and decrease the risk of exposure. These restrictions, in combination with home-stay arrangements and the onset of economic recession. As a result of public policies, financial difficulties and patient fear, many radiology departments have suffered a significant reduction in diagnostic examinations with important implications for their economic stability. The aim of this work is to evaluate the economic impact of the COVID-19 pandemic in the Radiology Department of an infectious disease hospital.
\end{abstract}

Keywords: COVID-19; Diagnostic imaging; Chest; CT-scan; Radiation Dose

\section{Introduction}

In Italy, the first case of SARS-CoV-2 infection (causing the systemic disease named COVID19) was officially reported on 20.02.2020. Since then the disease has evolved rapidly, causing a public health emergency not only in Italy but worldwide. The rapid growth in the number of patients with COVID-19, has revolutionized the hospital management, especially among the intensive care units, conditioning the daily multidisciplinary work of those health facilities most involved in the care of COVID-19. To limit the spread of the disease and create additional hospitalization and staffing capacities, many hospitals have closed wards and outpatients, postponing or cancelling hospitalizations, visits, examinations and services performed in election. Although the reduction in outpatient revenues and elective 
admissions may be partially offset by increased bed occupancy, new intensive care units and recovery of health care after the pandemic, this may not be sufficient. Some hospitals may have increased revenue from COVID-19 hospital admissions as well as higher personnel and additional resources costs, while other hospitals will suffer mostly loss of revenue because of state provisions to minimise non-essential services (1).

The COVID-19 pandemic caused an unprecedented effect on national radiological investigations. Since the World Health Organization officially declared the COVID-19 as a global pandemic, health policies have been rapidly organized to limit the spread of the virus and decrease the risk of exposure. These restrictions, in combination with homestay arrangements and the onset of economic recession, led to a sharp reduction in the demand for benefits (2) and loss diagnosis in primary and follow up diseases especially in oncologic patients

To reduce the risk of exposure and transmission to healthcare professionals and patients and to minimize contamination of equipment during COVID-19 patient transportation, our Radiology Department followed specific guidelines for an appropriate use of imaging diagnostic equipment (3-4)

All non-urgent procedures, if possible, have been postponed in patients with confirmed or suspected COVID-19. In particular, the use of chest CT in patients with COVID-19 was suggested for symptomatic hospitalized patients with specific clinical indications.

As a result of public policies, financial difficulties and patient fear, many radiology departments have suffered a significant reduction in diagnostic examinations with important implications for their economic stability.

In order to prepare a short- and long-term response to the COVID-19 crisis, objective data are needed to understand the magnitude of the reduction in activity volumes and imaging modes.

\section{Materials and Methods}

The National Institute of Infectious Diseases "L. Spallanzani" in Rome admitted the first two cases of COVID-19 patients in Italy on 20.02.2020. Subsequently the whole institute was progressively completely dedicated to the treatment of patients affected by COVID-19, so far. The execution of the radiological examinations during the pandemic has regulated by internal protocols on the appropriateness of the examinations, on the procedures of isolation of the patients and sanitization of the halls and equipment. There were no changes in the organic endowment of radiologists and medical personnel during the periods under examination. Access to external patients was allowed in a modulated manner in relation to the various stages of the pandemic in Italy. The access at the CT room was reserved only for COVID-19 patients, hospitalized or discharged and in follow-up.

We evaluated the impact of the COVID-19 pandemic on the activity volumes of our imaging department comparing a time range of 9 months (from March to December) within 2 years (2019 vs 2020), considering potential distortions due to monthly and 
seasonal variations as a limitation of the study. It is clear that the data on workloads are crucial to understand how radiologists respond to technical, professional and economic changes in a clinical practice and in an emergency setting. Traditionally, we have relied on the numbers of hours worked (although an economist would correctly use them as an indicator of resources invested rather than productivity) or the numerical count of exams. The progressive technological evolution introduced increasingly complex and expensive radiological equipments. The consequence is that the total number of examinations carried out does not reflect the actual workload on radiologists anymore (5-8).

For this reason, activity data were aggregated into homogeneous groups (no. of traditional radiology examinations, ultrasound, CT or MRI, interventional procedures) that were then evaluated according to four systems:

1. Numerical count of radiological examinations produced by the service.

2. Economic value based on the regional tariff system of the Lazio Region.

3. Method proposed by SNR-SIRM in 1998 and subsequent modifications (9-10).

It is characterized by the weight/exam parameter. Productivity is then determined on the basis of the number of points produced

4. Method based on work-RVU (Relative Value Unit), used in the USA, aimed mainly at the reimbursement of radiological services by government insurance but used in the literature for the assessment of radiological productivity. Productivity is then determined by the number of points produced (11).

To assess the complexity of examinations, the difficulty index expressed by the ratio of work-RVU/examinations and SNR points/examinations was also evaluated (1011).

We then proceeded to differentiate the data obtained between inpatients and outpatients, and analyzed in particular the data related to intensive care and examinations performed at the bedside of patients in the ward.

Further analysis was sustained on the comparison of costs incurred by the radiology department during the periods under review to assess the overall economic impact of the outbreak (12-14).

\section{Results}

\subsection{Production}

Numerical Count; total exams: 25,963 radiology exams were performed in 2019, 10,164 in 2020 (-61\%); the largest decrease was in bone densitometry (-89\%) followed by traditional radiology (-63\%), ultrasound (-72\%), MRI (-60\%), and CT (-14\%) (Fig 1). 


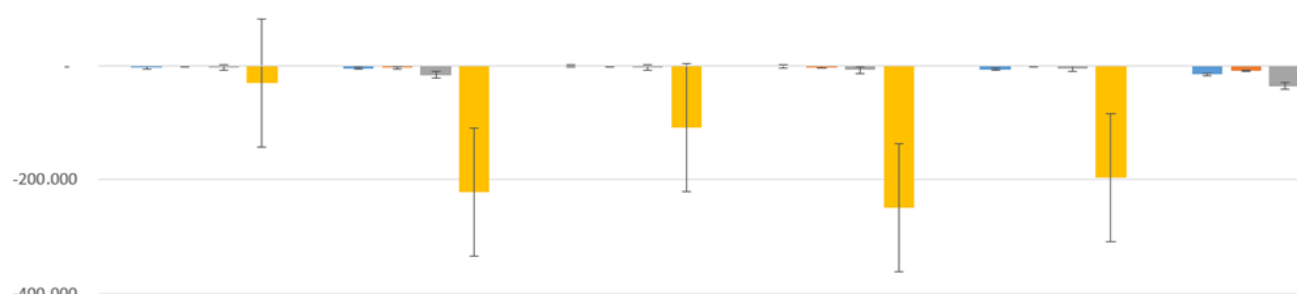

$-400.000$

$-600.000$

$-800.000$

\begin{tabular}{|c|c|c|c|c|c|c|}
\hline-1.000 .000 & $R x$ & Ultrasound & CT & MRI & MOC & TOTAL \\
\hline Exams number & -2.904 & -4.531 & -786 & -1.478 & -6.100 & -15.799 \\
\hline - WORK rwu & $-619,8$ & $-3829,9$ & $-1074,3$ & $-2405,1$ & $-1506,5$ & $-9435,6$ \\
\hline IIIRM & $-2904,0$ & $-15858,5$ & $-3379,8$ & $-7833,4$ & $-5490,0$ & $-35465,7$ \\
\hline II EURO & -30.407 & -220.694 & -108.107 & -249.500 & -195.281 & -803.990 \\
\hline
\end{tabular}

Fig 1 Comparison between radiological examinations performed in 2019 and 2020

\subsubsection{Internal Patients:}

In 2019, 6,515 radiological examinations were performed to internal patients, in 2020 $6,284(-4 \%)$ the largest decrease involved bone densitometry (-90\%) followed by ultrasound (-61\%), traditional radiology (-47\%), MRI (-29\%) while documenting an increase in CT examinations (+74\%) (Fig 2). 
2020 - 2019 VARIATION INPATIENTS

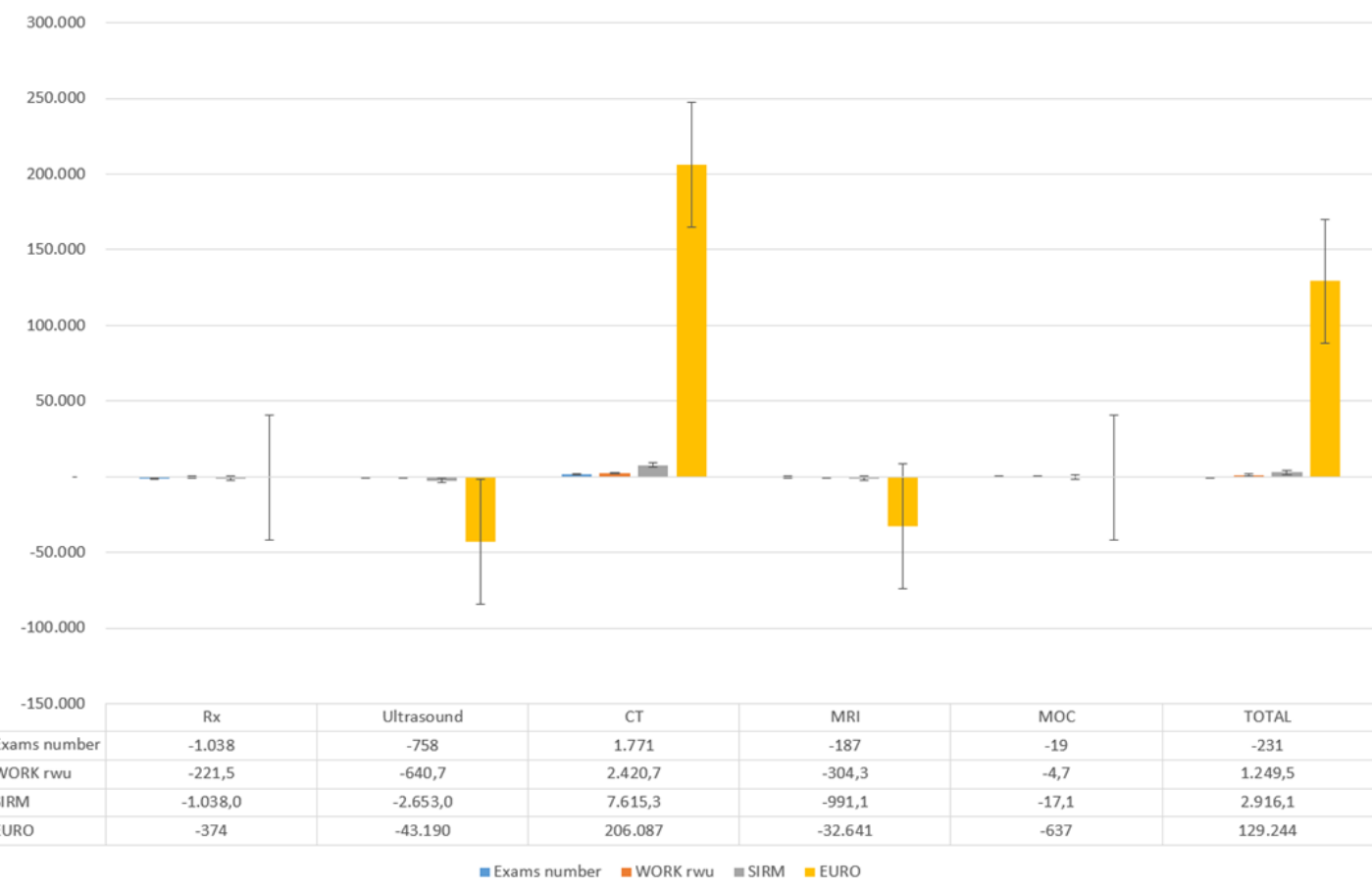

Fig 2 Comparison between internal patient's radiological examinations performed in 2019 and 2020.

\subsubsection{Outpatients:}

In 2019, 19,448 radiology examinations were performed for outpatients, in 2020 3,880 ($61 \%)$; the largest decrease was in bone densitometry $(-89 \%)$ followed by traditional radiology (-77\%), CT (-76\%), ultrasound (-75\%), MRI (-70\%) (Fig 3).

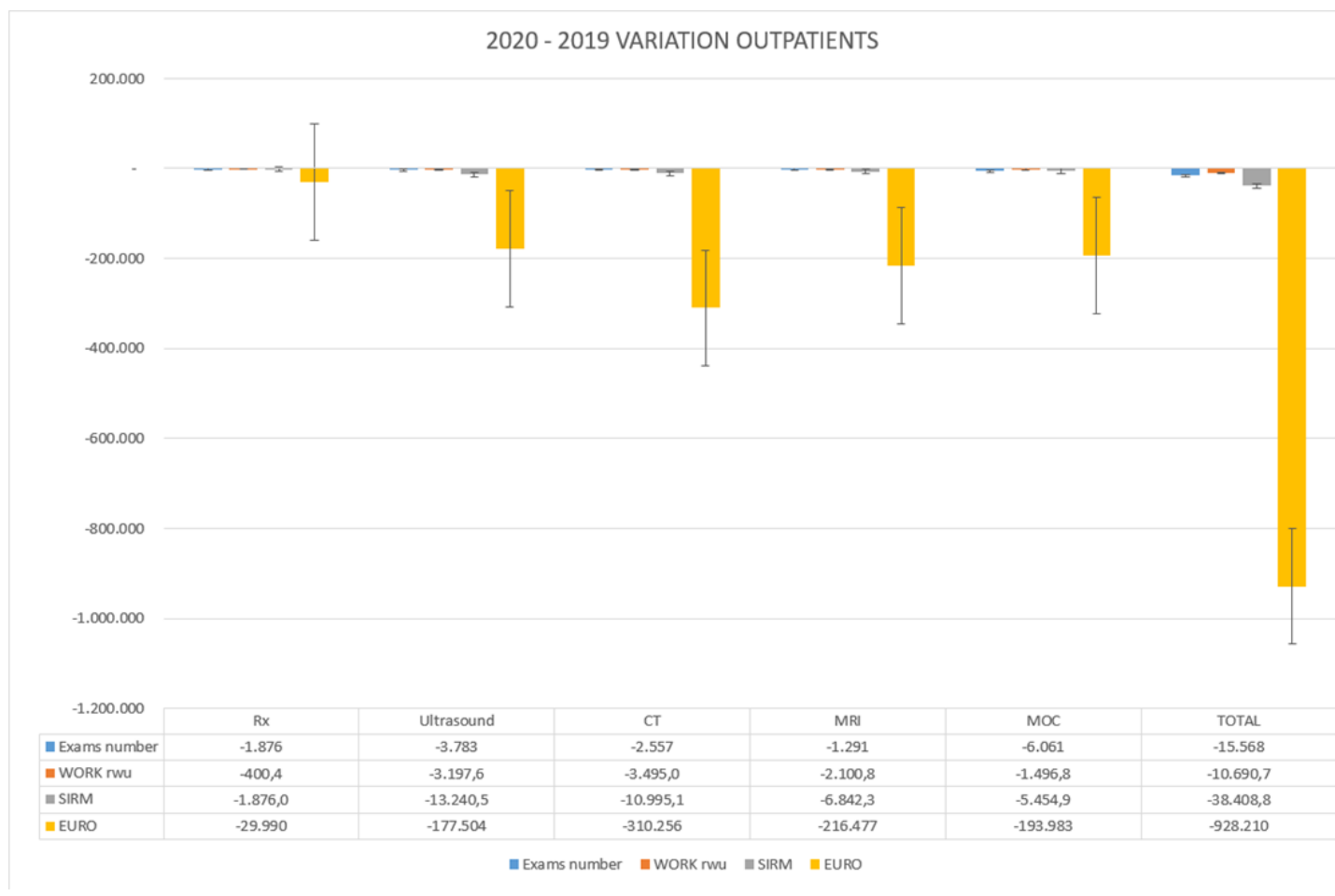


Fig 3 Comparison between external patient's radiological examinations performed in 2019 and 2020.

\subsection{Economic evaluation in Euros}

In the comparison between 2019 and 2020, the overall turnover of our department decreased by $47.2 \%$; the largest decrease involved bone densitometry (-89.57\%), followed by ultrasound (-70.85\%), MRI (-57.76\%) traditional radiology $(-39.63 \%)$, and CT $(-16.25 \%)$. In the analysis of the data by patient type, the total revenue for radiological examinations to internal patients, also evaluated for the periods 2019 and 2020, increased overall by $26.09 \%$, with decreases involving bone densitometry ($91.00 \%)$, ultrasound (-62.32\%), MRI (-26.55\%), and traditional radiology $(-1.07 \%)$, offset by an increase for CT examinations $(+77.07 \%)$. The same parameters evaluated for radiology activity to outpatients documented a $77.16 \%$ decrease in revenue; the largest decrease involved bone densitometry (-89.53\%) followed by CT (-78.77\%), ultrasound $(-73.30 \%)$, traditional radiology $(-72.01 \%)$ and MRI $(-70.15 \%)$.

Evaluating the overall activities with SIRM-SNR and work-RVU scores, we note 70,562.40 SIRM-SNR points in 2019 versus 35,096.70 in 2020 with a decrease of 50.26\% and 19,853.10 work-RVU points in 2019 and 10,417.53 in 2020 with a decrease of $47.53 \%$. In the evaluation of radiological activity for internal patients in 2019 20,248.8 SIRMSNR points were calculated, in $202023,164.9$ with an increase of $14.40 \%$. In the workRVU calculation we note 5,833.16 points in 2019 and 7,082.63 in 2020 with an increase of $21.42 \%$. In the evaluation of radiological activity for external patients in 2019 50,340.6 SIRM-SNR points were calculated, in 2020 11,931.8 with a decrease of $76.30 \%$. In the work-RVU calculation we note 14,025.58 points in 2019 and 3,334.9 in 2020 with a decrease of $76.22 \%$.

\subsubsection{Specific workload for intensive care/resuscitation departments.}

In 2019808 radiological examinations were performed, in 20201186 (+ 47\%); the largest increase involved CT $(+88 \%)$ followed by traditional radiology $(+82 \%)$, MRI $(+17 \%)$ only decrease ultrasound (-47\%). In 2019 2,567.80 SIRM-SNR points were calculated, in $20203,651.30(+42.20 \%)$. In 2019, 731.18 Work-RVU points were calculated, in 2020 1,082.84 (+48.09\%).

3.2.2. Urgent examinations in the department: traditional radiology and ultrasound

In 2019, 231 examinations were performed in the traditional radiology and ultrasound department, in 2020381 (+65\%); the largest increase was in ultrasound 37 examinations vs 0 , followed by traditional radiology (+49\%). In 2019, 231.00 SIRM-SNR points were calculated, in 2020473.50 (+104.98\%). In 2019, 49.30 work-RVU points were calculated, in 2020104.69 (+112.36\%). Finally, in the evaluation of changes in the incidence of examinations in urgency/emergency in 2019 3,308 radiological examinations were performed in urgency, in 2020 4,762 (+44\%); the largest decrease involved ultrasound $(-32 \%)$, followed by traditional radiology $(-10 \%)$, the largest increase was in CT $(+139 \%)$ and MRI $(+36 \%)$.

In 2019, 9,830.00 SIRM-SNR points were calculated, in 2020 16,693.50 (+69.82\%). In 2019, 2,802.59 Work-RVU points were calculated, in 2020 5,053.80 (+80.33\%). 
Finally, for the evaluation of the difficulty index calculated by the ratio of work-RVU points/exams for total exams in 2019 a value of 0.76 , in 2020 of $1.02(+34.04 \%)$ was calculated; For internal exams in 2019 a value of 0.90, in 2020 of $1.13(+25.88 \%)$ was calculated; For external exams in 2019 a value of 0.72, in 2020 of $0.86(+19.18 \%)$ was calculated. Evaluating the difficulty index from the SNR-SIRM points/exams ratio for total exams in 2019 a value of 2.72 was calculated, in 2020 a value of $3.45(+27.05 \%)$. For internal exams in 2019, a value of 3.11 was calculated, in 2020 a value of $3.69(+18.61 \%)$. For external exams in 2019, a value of 2.59 was calculated, in 2020 a value of 3.08 $(+18.80 \%)$.

\subsection{Costs}

The overall cost of production in 2020 increased by $28.96 \%$ compared to the corresponding period in 2019. In the comparison of the same periods, the cost of medical personnel increased by $31.36 \%$, the cost of drugs (mainly contrast media) decreased by $38.69 \%$ as well as the cost of radiological medical devices, which decreased by $30.55 \%$. Other hospital costs transferred to the radiology department (non-radiological medical devices, internal hospital transport and other fixed costs) increased by $146.80 \%$. In the final operating result, there was a loss for the 2020 period that was approximately double the previous 2019 period.

\section{Discussion}

The data reported for our structure obviously have limitations related to our particular type, without ED and dedicated exclusively to the diagnosis and treatment of infectious diseases. In addition the number of annual global examinations of diagnostic imaging is conditioned by the presence of a single equipment for CT and MRI examinations and only two sections of diagnostic ultrasound mainly abdominal.

Literature data show that most of the revenue of a hospital radiology department comes from examinations of outpatients, while examinations of inpatients and emergencies account for about $20 \%$ and $27 \%$ of the total.

During the COVID-19 pandemic, all studies reported consistent and dramatic declines in all radiology examinations, with significant reductions in outpatient accesses of up to $70 \%$, while examining the various imaging techniques showed reductions of $56 \%$ for MR, 53\% for conventional radiology, and 47\% for CT [15-16]; in particular for outpatients, the most significant decreases peaked for mammography examinations (94\%), followed in descending order by nuclear medicine (85\%), MRI $(74 \%)$, ultrasound (64\%), interventional radiology (56\%), CT (46\%), and radiography $(22 \%)(17-18)$

One study tracked the trend in wRWUs documenting an average decline of $52 \%$ in the United States during the pandemic peak. By modality, CT and traditional radiography (including mammography) together accounted for well over half of the total practice wRVU decreases (19). 
An Italian study evaluated data over an 8-week period during the Italian lockdown (March 9-May 3, 2020) cancer center, it showed a reduction in volume of $26.0 \%$ for CT, $34.7 \%$ for MRI, $34.8 \%$ for X-ray and $58.5 \%$ for US (20)

Our experience confirms an overall decline in radiology examinations (-64\%), especially external examinations (-80\%) (the only exception being internal CT examinations which increased $+70 \%)$ resulting in a decline in revenue $(-47 \%)$, compounded by a marked increase in direct costs resulted in a heavy liability.

The pandemic has also changed our way of working with an increase in radiological examinations to be performed in the department $(+65 \%)$ (ultrasound and traditional radiology) and a marked commitment to meet urgent requests $(+44 \%)$ (especially CT $+139 \%$ ) of all departments and in particular intensive care units $(+47 \%)$.

The analysis of the workload obviously shows an almost superimposable overall decrease both analyzing the w-rvu (-47\%) and the SIRM-SNR points $(-50 \%)$, it should be noted that instead in the analysis of internal patients there is an increase in workloads respectively $+21 \%$ and $+14 \%$ (as opposed to the number of examinations) supported by a decrease in low-complexity examinations as confirmed by the increase of both complexity indices respectively $+25 \%$ and $+18 \%$.

This decrease in radiological activity has been related by some to multiple factors including the severity of the ongoing pandemic, social restrictions on population movement, patients' fears/concerns about leaving their homes and entering hospital facilities, delays in booking external radiological examinations during the acute phase of the pandemic, the impact of the economic recession on healthcare costs, and the type of radiological examination needed [21-22].

Certainly, this pandemic period has profoundly altered our work rhythms and diagnostic workflows. The economic loss of a Diagnostic Imaging department is evident and unavoidable especially considering the current economic parameters of remuneration of radiological activity.

This is even more evident in a Radiology department, like ours, dedicated exclusively to patients with infectious diseases and currently engaged only with COVID-19 patients.

Probably, as it has already been aired in other areas, it will be necessary to include correction factors in the management and economic evaluation of the patient with COVID-19 that not only always determines a slowdown of the normal workflow for the known problems of environmental isolation and equipment and sanitation but also determines a significant increase in operating costs of the department (23-25)

\section{Patents}

This section is not mandatory but may be added if there are patents resulting from the work reported in this manuscript.

Author Contributions: Conceptualization, CM. and VS; methodology, AP, FA and FDS; software, NF and SI; validation PC., writing-original draft preparation, MC; writing-review and editing, PC and VS.; supervision, SI. All authors have read and agreed to the published version of the manuscript. 
Acknowledgments: The authors are grateful to the COVID-19 team of Radiology Department at the Lazzaro Spallanzani National Institute of Infectious Diseases, Rome, for providing daily support in this critical situation: Ivan Berretta, Nicola Caretto, Simone Chiari, Marco Cellini, Felicia Coroian, Mirko Costa, Angela Maria Cuellar Imenez, Rosalia De Luca, Daniele Di Bartolomeo, Luigia Di Felice, Tiziana Fazio, Tiziano Fellone, Cristian Gaglio, Filomena Graziano, Chiara Iapalucci, Giulia Lattuille, Luisa Mari, Roberto Mengarelli, Maurizio Morea, Roberta Pappalardo, Valter Possanzini, Cristina Stornaiuolo, Maruska Tabacco, Natalia Tartaglia, Alessia Trombetti and COVID 19 INMI Study Group Maria Alessandra Abbonizio, Amina Abdeddaim, Chiara Agrati, Fabrizio Albarello, Gioia Amadei, Alessandra Amendola, Tommaso Ascoli Bartoli, Francesco Baldini, Raffaella Barbaro, Barbara Bartolini, Rita Bellagamba, Martina Benigni, Nazario Bevilacqua, Gianlugi Biava, Michele Bibas, Licia Bordi, Veronica Bordoni, Evangelo Boumis, Donatella Busso, Marta Camici, Paolo Campioni, Alessandro Capone, Cinzia Caporale, Emanuela Caraffa, Ilaria Caravella, Fabrizio Carletti, Concetta Castilletti, Adriana Cataldo, Stefano Cerilli, Carlotta Cerva, Roberta Chiappini, Pierangelo Chinello, Carmine Ciaralli, Stefania Cicalini, Francesca Colavita, Angela Corpolongo, Massimo Cristofaro, Salvatore Curiale, Alessandra D'Abramo, Cristina Dantimi, Alessia De Angelis, Giada De Angelis, Maria Grazia De Palo, Federico De Zottis, Virginia Di Bari, Rachele Di Lorenzo, Federica Di Stefano, Gianpiero D'Offizi, Davide Donno, Francesca Faraglia, Federica Ferraro, Lorena Fiorentini, Andrea Frustaci, Nicoletta Fusco, Matteo Fusetti, Vincenzo Galati, Roberta Gagliardini, Paola Gallì, Gabriele Garotto, Saba Gebremeskel Tekle, Maria Letizia Giancola, Filippo Giansante, Emanuela Giombini, Guido Granata, Maria Cristina Greci, Elisabetta Grilli, Susanna Grisetti, Gina Gualano, Fabio Iacomi, Giuseppina Iannicelli, Stefania Ianniello, Giuseppe Ippolito, Eleonora Lalle, Simone Lanini, Daniele Lapa, Luciana Lepore, Raffaella Libertone, Raffaella Lionetti, Giuseppina Liuzzi, Laura Loiacono, Andrea Lucia, Franco Lufrani, Manuela Macchione, Gaetano Maffongelli, Alessandra Marani, Luisa Marchioni, Andrea Mariano, Maria Cristina Marini, Micaela Maritti, Alessandra Mastrobattista, Giulia Matusali, Valentina Mazzotta, Paola Mencarini, Silvia Meschi, Francesco Messina, Annalisa Mondi, Marzia Montalbano, Chiara Montaldo, Silvia Mosti, Silvia Murachelli, Maria Musso, Emanuele Nicastri, Pasquale Noto, Roberto Noto, Alessandra Oliva, Sandrine Ottou, Claudia Palazzolo, Emanuele Pallini, Fabrizio Palmieri, Carlo Pareo, Virgilio Passeri, Federico Pelliccioni, Antonella Petrecchia, Ada Petrone, Carmela Pinnetti, Maria Pisciotta, Silvia Pittalis, Agostina Pontarelli, Costanza Proietti, Vincenzo Puro, Paolo Migliorisi Ramazzini, Alessia Rianda, Gabriele Rinonapoli, Silvia Rosati, Martina Rueca, Alessandra Sacchi, Alessandro Sampaolesi, Francesco Sanasi, Carmen Santagata, Alessandra Scarabello, Silvana Scarcia, Vincenzo Schininà, Paola Scognamiglio, Laura Scorzolini, Giulia Stazi, Fabrizio Taglietti, Chiara Taibi, Roberto Tonnarini, Simone Topino, Francesco Vaia, Francesco Vairo, Maria Beatrice Valli, Alessandra Vergori, Laura Vincenzi, Ubaldo Visco-Comandini, Pietro Vittozzi, and Mauro Zaccarelli.

Conflicts of Interest: The authors declare no conflict of interest.

\section{References}

1) Kwee TC: KweeRM. Chest CT in COVID-19: What the Radiologist Needs to Know RadioGraphics Vol. 40, No. 7 Published Online: Oct 232020 https://doi.org/10.1148/rg.2020200159

2) Albarello F, Pianura E, Di Stefano F, Cristofaro M, Petrone A, Marchioni L, Palazzolo C, Schininà V, Nicastri E, Petrosillo N, Campioni P, Eskild P, Zumla A, Ippolito G. 2019-novel Coronavirus severe adult respiratory distress syndrome in two cases in Italy: An uncommon radiological presentation. Int J Infect Dis. 2020 Apr; 93:192-197. doi: 10.1016/j.ijid.2020.02.043.

3) Klok FA, Kruip MJHA, van der Meer NJM, et al. Confirmation of the high cumulative incidence of thromboticcomplications in critically ill ICU patients with COVID-19: An updated analysis. Thromb Res 2020; 191:148-150

4) Grillet F, Behr J, Calame P, Aubry S, Delabrousse E. Acute Pulmonary Embolism Associated with COVID-19 Pneumonia Detected with Pulmonary CT Angiography. Radiology 2020;296(3):E186-E188.

5) Kaminetzky M, Moore W, Fansiwala K, et al. Pulmonary embolism on CTPA in COVID-19 patients. Radiol CardiothoracImaging 2020; 2(4):e200308

6) Oudkerk M, Büller HR, Di Kuijpers, van Es N, Oudkerk SF, McLoud TC, Gommers D, J Van Diagnosis, Prevention, and Treatment of Thromboembolic Complications in COVID-19: Report of the National Institute for Public Health of the Netherlands Radiology Vol. 297, No. 1 Published Online:Apr 232020 https://doi.org/10.1148/radiol.2020201629

7) Gerald C, Jane J L, Adeel J, Vamsikrishna G, Homa N, Sahar MM, Fahimehalsadat S, Jolanta M Venous Thromboembolism among Hospitalized Patients with COVID-19 Undergoing Thromboprophylaxis: A Systematic Review and Meta-AnalysisJ Clin Med. 2020 Aug 3;9(8):2489. doi: 10.3390/jcm9082489. 
8) Zhong L. Analysis of Chest CT Results of Coronavirus Disease 2019 (COVID-19) Patients at First Follow-Up. Can Respir J. 2020 Nov 1;2020:5328267. doi: 10.1155/2020/5328267. eCollection 2020.

9) Kang Z, X Li X, Zhou S Recommendation of low-dose CT in the detection and management of COVID-2019Eur Radiol 2020 Aug; 30(8):4356-4357. doi: 10.1007/s00330-020-06809-6.

10) Pan F, Ye T, Sun $P$ et al. Time course of lung changes on chest CT during recovery from 2019 novel coronavirus (COVID-19) pneumonia. Radiology. 2020 doi.org/10.1148/radiol.2020200370:200370

11) Tao G, Guangbin W, Jianwen W, Xinfu G Timely Diagnosis and Treatment Shortens the Time to Resolution of Coronavirus Disease (COVID-19) Pneumonia and Lowers the Highest and Last CT Scores From Sequential Chest CTAJR 2020; 215:1-7 ISSNL 0361-803X/20/2152-1

12) Bernheim A, Mei X, Huang M, Yang Y, Fayad ZA, Zhang N, Diao K, Lin B, Zhu X, Li K, Li S, Shan H, Jacobi A, Chung M. Chest CT Findings in Coronavirus Disease-19 (COVID-19): Relationship to Duration of Infection. Radiology. 2020 Jun; 295 (3):200463. doi: 10.1148/radiol.2020200463

13) Busi Rizzi E, Schinina V, Gentile FP, Bibbolino C. Reduced computed tomography radiation dose in HIV-related pneumonia: effect on diagnostic image quality Clinical Imaging 31 2007, 178-184

14) Cristofaro M, Busi Rizzi E, Piselli P, Pianura E, Petrone A, Fusco N, Di Stefano F, Schinina' V. Image quality and radiation dose reduction in chest CT in pulmonary infection. Radiol Med. 2020 May;125(5):451-460. doi: 10.1007/s11547-020-01139-5.

15) Mayo-Smith WW, Hara AK, Mahesh M, Sahani DV, Pavlicek W How I Do It: Managing Radiation Dose in CTRadiology: Vol 273: Number, December 2014 Published Online:Nov 242014 doi.org/10.1148/radiol.14132328

16) Smith-Bindman R, Wang Y, Chu P, Chung R, Einstein AJ, Balcombe J, Cocker M, Das M, Delman BN, Flynn M, Gould R, Lee RK, Yellen-Nelson T, Schindera S, Seibert A, Starkey J, Suntharalingam S, Wetter A, Wildberger JE, Miglioretti DL. International variation in radiation dose for computed tomography examinations: prospective cohort study. BMJ. 2019 Jan 2; 364:k4931. doi: 10.1136/bmj.k4931.

17) Lumbreras B, Salinas JM, Gonzalez-Alvarez I. Cumulative exposure to ionising radiation from diagnostic imaging tests: a 12year follow-up population-based analysis in Spain BMJ Open. 2019 Sep 18;9(9): e030905. doi: 10.1136/bmjopen-2019-030905.

18) Stopsack KH, Cerhan JR. Cumulative Doses of Ionizing Radiation From Computed Tomography: A Population-Based Study Mayo Clin Proc 2019 Oct;94(10):2011-2021. doi: 10.1016/j. mayocp. 2019.05.022.

19) Rubin GD, Ryerson CJ, Haramati LB, Sverzellati N, Kanne JP, Raoof S, Schluger NW, Volpi A, Yim JJ et all. The Role of Chest Imaging in Patient Management during the COVID-19 Pandemic: A Multinational Consensus Statement from the Fleischner Society RadiologyVol. 296, No. 1 Published Online:Apr 2020 doi.org/10.1148/radiol. 2020201365

20) Kalra MK, Homayounieh F, Arru C, Holmberg O, Vassileva J. Chest CT practice and protocols for COVID-19 from radiation dose management perspective Eur Radiol. 2020 Dec; 30(12):6554-6560. doi: 10.1007/s00330-020-07034-x.

21) Homayounieh F, Kalra MK. COVID-19 and chest CT: protocol and dose optimization, Webinar 9 April 2020 of International Atomic Energy Agency, http://ns-files.iaea.org/video/rpop-webinar-apr2020

22) Hiroaki S, Mari I, Lin S, Wataru F, Chiemi S, Yoshihiro M, Takafumi I, Tomoyuki A, Morihito O, Kazuo Awai, Satoshi T Biological Effects of Low-Dose Chest CT on Chromosomal DNA. Published Online:Mar 102020 https://doi.org/10.1148/radiol.2020190389

23) Hauptmann M, Daniels RD, Cardis E et al. Epidemiological Studies of Low-Dose Ionizing Radiation and Cancer: Summary Bias Assessment and Meta-Analysis. JNCI Monographs. 2020 (56):188-200.

24) Mohammad AR - Charbel S. Radiation dose reduction considerations and imaging patterns of ground glass opacities in coronavirus: risk of over exposure in computed tomography. La radiologia medica doi.org/10.1007/s11547-020-01271-2

25) Homayounieh F, Holmberg O, Umairi RA, Aly S, Basevičius A et all. Variations in CT Utilization, Protocols, and Radiation Doses in COVID-19 Pneumonia: Results from 28 Countries in the IAEA Study Radiology Published Online:Nov 10 2020https://doi.org/10.1148/radiol.2020203453

26) Lee C. Managing Radiation Dose from Chest CT in COVID-19 Patients Published Online: Radiology Nov 102020 https://doi.org/10.1148/radiol.2020204129

27) Ameri A, Ameri P, Rahnama N, Mokhtari M, Sedaghat M, Hadavand F, Bozorgmehr R, Haghighi M, Taghizadeh-Hesary F. Low-dose Whole-lung Irradiation for COVID-19 Pneumonia: Final Results of a Pilot Study. Int J Radiat Oncol Biol Phys. 2020 Dec 2:S0360-3016(20)34605-8. doi: 10.1016/j.ijrobp.2020.11.065.

28) Sanmamed N, Alcantara P, Cerezo E, Gaztañaga M, Cabello N, Gómez S, Bustos A, Doval A, Corona J, Rodriguez G, Duffort M, Ortuño F, de Castro J, Fuentes M, Sanz A, López A, Vazquez M. Low dose radiotherapy in the management of covid19 pneumonia (LOWRAD-Cov19). Preliminary report. Int J Radiat Oncol Biol Phys. 2020 Nov 26:S0360-3016(20)34571-5. doi: 10.1016/j.ijrobp.2020.11.049. 
29) Prasanna PG, Woloschak GE, DiCarlo AL, Buchsbaum JC, Schaue D, Chakravarti A, Cucinotta FA, Formenti SC, Guha C, Hu DJ, Khan MK, Kirsch DG, Krishnan S, Leitner WW, Marples B, McBride W, Mehta MP, Rafii S, Sharon E, Sullivan JM, Weichselbaum RR, Ahmed MM, Vikram B, Coleman CN, Held KD. Low-Dose RadiationTherapy (LDRT) for COVID-19: Benefits or Risks? Radiat Res. 2020 Nov 10;194(5):452-464. doi: 10.1667.

30) Mortazavi A, Mortazavi SMJ, Sihver L. Selective Pressure-Free Treatments for COVID-19. Radiation 2021, 1, 18-32; doi:10.3390/radiation1010003

31) Li, J., Wang, X., Huang, X. et al. Application of Care Dose $4 \mathrm{D}$ combined with Karl 3D technology in the low dose computed tomography for the follow-up of COVID-19. BMC Med Imaging 20, 56, 2020. https://doi.org/10.1186/s12880-020-00456-5 